\title{
Inductive limit of operators and its applications
}

by

J. JANAS (Kraków)

\begin{abstract}
The paper deals mainly with spectral properties of inductive limits of operators in Hilbert spaces. Applications to inductive limits of Riesz and hyponormal as well as to other operators are also given. In particular, extensions of Marchenko theorems to normal operators are shown.
\end{abstract}

Notation. All Hilbert spaces considered in the following are complex. For a complex Hilbert space $H$ with a scalar product $(\cdot, \cdot), L(H)$ denotes the space of all linear and bounded operators on $H$. If $T$ is a closed densely defined operator in $H$, then $\sigma(T)$ (respectively $\sigma_{\pi}(T)$ ) stands for the spectrum of $T$ (respectively the approximate point spectrum of $T$ ) and $D(T)$ for the domain of $T$.

1. Let us recall the notion of inductive limit of Hilbert spaces. Suppose we are given a sequence of Hilbert spaces $H_{k}, k=1,2, \ldots$ We say that a Hilbert space $H$ is an inductive limit of the $H_{k}$ if there are isometries $\gamma_{k}^{l}: H_{k}$ $\rightarrow H_{l}(k \leqslant l)$ and $\gamma_{k}: H_{k} \rightarrow H$ such that the following conditions are satisfied:

(a) $\gamma_{k}^{k}$ is the identity on $H_{k}$.

(b) $\gamma_{k}^{m}=\gamma_{l}^{m} \circ \gamma_{k}^{l}$ if $k \leqslant l \leqslant m$.

(c) $\gamma_{k}=\gamma_{l} \circ \gamma_{k}^{l}$ if $k \leqslant l$.

(d) $H=\bigvee_{k \geqslant 1} \gamma_{k} H_{k}$.

By the above definition there is no loss of generality in denoting by $\|\cdot\|$ the norm in every $H_{k}$ and also in $H$. We write $H=\lim _{\gamma} H_{k}$.

The starting point of our work concerns a generalization of a result of Marchenko [3]. Namely, for a given sequence of selfadjoint operators $A_{n}$ in $H_{n}$ he has found a sufficient condition for the essential selfadjointness of the operator

$$
A_{\infty} \varphi=\lim _{m \rightarrow \infty} \gamma_{m} A_{m} \gamma_{n}^{m} \varphi_{n}, \quad \varphi=\gamma_{n} \varphi_{n},
$$

on $D_{\infty}=\bigcup_{n} \gamma_{n} D_{n}$, where $D_{n}=$ the domain of $A_{n}$. It turns out that a similar condition imposed on general closed densely defined operators $L_{k}$ in $H_{k}$ also 
guarantees the existence of

$$
L_{\infty} \varphi=\lim _{m \rightarrow \infty} \gamma_{m} L_{n} \gamma_{n}^{m} \varphi_{n}, \quad \varphi=\gamma_{n} \varphi_{n},
$$

on $D_{\infty}=\bigcup_{n} \gamma_{n} D_{n}$, where $D_{n}=$ the domain of $L_{n}$. To be more precise, suppose that the above sequence $L_{n}$ satisfies the following condition:

$$
\gamma_{n}^{n+1} D_{n} \subset D_{n+1}, \quad \gamma_{n}^{n+1} D_{n}^{*} \subset D_{n+1}^{*},
$$

where $D_{n}^{*}=$ the domain of $L_{n}^{*}$.

THEOREM 1.1. Let $L_{n}$ be a sequence of densely defined closable operators in $H_{N}$ with domains $D_{N}$ satisfying the above condition (i). Assume that for any $\varepsilon>0$ there exists $n_{0}(\varepsilon)$ such that for every $m>n \geqslant n_{0}(\varepsilon)$ and any $\varphi \in D_{n}, \psi \in D_{n}^{*}$

$(*)$

$$
\left\|\left(L_{m} \gamma_{n}^{m}-\gamma_{n}^{m} L_{n}\right) \varphi\right\| \leqslant \varepsilon\left(\|\varphi\|+\left\|L_{m} \gamma_{n}^{m} \varphi\right\|+\left\|L_{n} \varphi\right\|\right),
$$

$$
\left\|\left(L_{m}^{*} \gamma_{n}^{m}-\gamma_{n}^{m} L_{n}^{*}\right) \psi\right\| \leqslant \varepsilon\left(\|\psi\|+\left\|L_{m}^{*} \gamma_{n}^{m} \psi\right\|+\left\|L_{n}^{*} \psi\right\|\right) .
$$

Then equality (1) defines a closable densely defined operator $L_{\infty}$ on $D_{\infty}$. Moreover, for any $\psi \in D_{\infty}^{*}=\bigcup_{n} \gamma_{n} D_{n}^{*}$ the limit

$$
A_{\infty} \psi \stackrel{\mathrm{df}}{=} \lim _{m \rightarrow \infty} \gamma_{m} L_{m}^{*} \gamma_{n}^{m} \psi_{n}, \quad \psi=\gamma_{n} \psi_{n},
$$

exists and $A_{\infty} \subset L_{\infty}^{*}$.

Proof. Let $\varphi=\gamma_{n} \varphi_{n}, \psi=\gamma_{n} \psi_{n}$. The existence of $\lim _{m \rightarrow \infty} \gamma_{m} L_{m} \gamma_{n}^{m} \varphi_{n}$ $=L_{\infty} \varphi$ and $\lim _{m \rightarrow \infty} \gamma_{m} L_{m}^{*} \gamma_{n}^{m} \psi_{n}=A_{\infty} \psi$ can be proved exactly in the same way as for selfadjoint operators [3].

Now we will show that $L_{\infty}$ is closable on $D_{\infty}$. First note that $\bar{D}_{\infty}=H$ and so $L_{\infty}^{*}$ exists. Moreover, we have $D_{\infty}^{*} \subset D\left(L_{\infty}^{*}\right)$.

Indeed, for $\varphi=\gamma_{n} \varphi_{n} \in D_{\infty}$ and $\psi=\gamma_{s} \psi_{s} \in D_{\infty}^{*}$ we write (with $m>s$ and $m>n)$

$$
\begin{aligned}
& \left(\gamma_{n} \varphi_{n}, \gamma_{m} L_{m}^{*} \gamma_{s}^{m} \psi_{s}\right)=\left(\gamma_{m} \gamma_{n}^{m} \varphi_{n}, \gamma_{m} L_{m}^{*} \gamma_{s}^{m} \psi_{s}\right)=\left(\gamma_{n}^{m} \varphi_{n}, L_{m}^{*} \gamma_{s}^{m} \psi_{s}\right) \\
& =\left(L_{m} \gamma_{n}^{m} \varphi_{n}, \gamma_{s}^{m} \psi_{s}\right)=\left(\gamma_{m} L_{m} \gamma_{n}^{m} \varphi_{n}, \gamma_{m} \gamma_{s}^{m} \psi_{s}\right)=\left(\gamma_{m} L_{m} \gamma_{n}^{m} \varphi_{n}, \gamma_{s} \psi_{s}\right)
\end{aligned}
$$

Hence

$$
\left(\varphi, A_{\infty} \psi\right)=\lim _{m \rightarrow \infty}\left(\gamma_{n} \varphi_{n}, \gamma_{m} L_{m}^{*} \gamma_{s}^{m} \psi_{s}\right)=\lim _{m \rightarrow \infty}\left(\gamma_{m} L_{m} \gamma_{n}^{m} \varphi_{n}, \gamma_{s} \psi_{s}\right)=\left(L_{\infty} \varphi, \psi\right)
$$

and so $\psi \in D\left(L_{\infty}^{*}\right)$.

Now applying a result of von Neumann [4] we know that $L_{\infty}$ is closable (and $L_{\infty}=L_{\infty}^{* *}$ ). The inclusion $A_{\infty} \subset L_{\infty}^{*}$ is also evident by the above equalities.
Remark 1.2. If $L_{n} \in L\left(H_{n}\right)$ and if the norms $\left\|L_{n}\right\|$ are uniformly bounded and satisfy condition $(*)$ then $L_{\infty}$ can' be extended to a bounded operator $L$ on $H$.

The operator $\bar{L}_{\infty} \stackrel{\mathrm{d} f}{=} L$ shares some properties of the approximating sequence $L_{n}$. For example: if the $L_{n}$ are symmetric (hyponormal) then $L$ is also symmetric (hyponormal). Here $T$ is called hyponormal if $T$ is densely defined, closed, $D(T) \subset D\left(T^{*}\right)$ and $\left\|T^{*} x\right\| \leqslant\|T x\|, x \in D(T)$. (We do not assume that $D(T)=D\left(T^{*}\right)$, the condition required in [4]).

The proofs are straightforward and left to the reader.

Remark 1.3. Theorem 1.1 has an obvious extension to linear mappings $L_{n}: H_{n} \rightarrow K_{n}$ (closed, densely defined) between Hilbert spaces $H_{n}, K_{n}$. Conditions $(*)$ and $(* *)$ also have natural interpretation in this context. In particular, (*) is trivially satisfied if $L_{n+1} \gamma_{n}^{n+1}=\tau_{n}^{n+1} L_{n}$, where $\tau_{n}^{n+1}: K_{n}$ $\rightarrow K_{n+1}$ denotes an isometric embedding.

Now we will give a few applications (examples) of Theorem 1.1.

EXAMPLE 1.4. Let $A_{i}$ be a sequence of closed densely defined operators in $\boldsymbol{H}_{i}$, a Hilbert space with norm $\|\cdot\|_{i}$. Suppose we are given a sequence $e_{i} \in D\left(A_{i}\right) \cap D\left(A_{i}^{*}\right),\left\|e_{i}\right\|_{i}=1$, such that

$$
\sum_{i}\left\|A_{i} e_{i}\right\|_{i}<+\infty, \quad \sum_{i}\left\|A_{i}^{*} e_{i}\right\|_{i}<+\infty
$$

Then the expression

$$
\sum_{i} I_{1} \otimes \ldots \otimes I_{i-1} \otimes A_{i} \otimes I_{i+1} \otimes \ldots
$$

where $I_{i}$ denotes the identity on $\boldsymbol{H}_{i}$, has a meaning and defines a closable densely defined operator in the infinite tensor product $H=\bigotimes_{1\left(e_{i}\right)}^{\infty} H_{i}$ with the stabilizing sequence $\left(e_{i}\right)$.

Here to apply Theorem 1.1 we put

$$
\begin{gathered}
L_{n}=\sum_{1}^{n} I_{1} \otimes \ldots \otimes I_{i-1} \otimes A_{i} \otimes I_{i+1} \otimes \ldots \otimes I_{n}, \\
H_{n}=\otimes_{1}^{n} H_{i}, \quad \gamma_{n}^{n+1} f=f \otimes e_{n+1} .
\end{gathered}
$$

Conditions (*) and (**) of Theorem 1.1 can be verified, using $(\alpha)$, in the same way as in [3] for selfadjoint operators.

EXAMPLe 1.5 (a problem). Let $\boldsymbol{R}_{j n}: L^{2}\left(\boldsymbol{R}^{n}\right) \rightarrow L^{2}\left(\boldsymbol{R}^{n}\right), 1 \leqslant j \leqslant n$, be the Riesz transforms. Set $d \mu_{n}=\alpha_{n}|x|^{-n} d x, \alpha_{n}=\Gamma(n / 2) / \pi^{n / 2}$. A straightforward reasoning shows that $L_{n}=R_{1 n}$ is a densely defined closable operator in 
$L^{2}\left(\mu_{n}\right)$. By a result of [12] there exists a unique (up to a constant) measure $\mu$ on the Borel subsets of $\boldsymbol{R}^{\infty}=\boldsymbol{R} \times \boldsymbol{R} \times \ldots$ such that:

(a) $\mu(\lambda E)=\mu(E), \lambda>0$.

(b) $\mu(U E)=\mu(E)$ for every unitary $U: l^{2} \rightarrow l^{2}$ which maps $\boldsymbol{R}_{0}^{\infty} \rightarrow \boldsymbol{R}_{0}^{\infty}$, where $\boldsymbol{R}_{0}^{\infty}$ denotes the real sequences with all but a finite number of coordinates different from zero.

(c) If $p_{n}: \boldsymbol{R}^{\infty} \rightarrow \boldsymbol{R}^{n}$ is the canonical projection, then for any $E$ $=p_{n}^{-1}\left(E_{n}\right), \mu(E)=\mu_{n}\left(E_{n}\right)$

We claim that the $L_{n}$ satisfy condition (*) of Theorem 1.1, where

$$
\gamma_{n}^{n+1}: f \rightarrow f, \quad \gamma_{n}: f \rightarrow f \in L^{2}(\mu) .
$$

It is easy to check that both above mappings are isometric. In fact, for $f \in L^{2}\left(\mu_{n}\right)$ we have

$$
\begin{aligned}
\left\|\gamma_{n}^{n+1} f\right\|^{2} & =\int|f|^{2} d \mu_{n+1}=\alpha_{n+1} \int_{\mathbb{R}^{n}}|f|^{2} \int_{-\infty}^{\infty} \frac{d x_{n+1} d x}{\left(|x|^{2}+x_{n+1}^{2}\right)^{(n+1) / 2}} \\
& =\int_{\mathbb{R}^{n}}|f|^{2} \frac{\sqrt{\pi}}{|x|^{n}} \frac{\Gamma\left(\frac{n}{2}\right)}{\Gamma\left(\frac{n+1}{2}\right)} \frac{\Gamma\left(\frac{n+1}{2}\right)}{\pi \frac{n+1}{2}} d x=\int|f|^{2} d \mu_{n}=\|f\|^{2} .
\end{aligned}
$$

It is clear that $L^{2}(\mu)=\lim _{\gamma} L^{2}\left(\mu_{n}\right)$. A careful reasoning shows that

$$
L_{n+1} \gamma_{n}^{n+1} \vec{f}=\gamma_{n}^{n+1} L_{n} f, \quad f \in L^{1}\left(\mu_{n}\right) \cap L^{2}\left(\mu_{n}\right) .
$$

It follows that

$$
L_{m} \gamma_{n}^{m} f=\gamma_{n}^{m} L_{n} f
$$

for any $m>n$, and so condition $(*)$ of Theorem 1.1 is trivially satisfied. In order to apply Th. 1.1 we should also verify condition $(* *)$ for $R_{1 n}^{*}$. Unfortunately, we were not able to prove or disprove this condition.

EXAMPLE 1.6. Suppose we are given a sequence $T_{i}$ of bounded operators in $H_{i}$. Theorem 1.1 can also be applied to obtain the well-known sufficient condition for the existence of the countable tensor product of the $T_{i}$. Assume that there exists a sequence $e^{(i)}$ of unit vectors, $\left\|e^{(i)}\right\|=1$, such that

$$
\sum_{i}\left\|e^{(i)}-T_{i} e^{(i)}\right\|<+\infty .
$$

Let $K=\otimes_{1\left(e^{(i)}\right)}^{\infty} H_{i}$ be the countable tensor product of the $H_{i}$ with the stabilizing sequence $\left(e^{(i)}\right)$. Let $K_{n}=\bigotimes_{1}^{n} H_{i}, \quad L_{n}=\bigotimes_{1}^{n} T_{i}$ and define $\gamma_{n}^{n+1}: K_{n} \rightarrow K_{n+1}, \gamma_{n}: K_{n} \rightarrow K$ by

$$
\gamma_{n}^{n+1} f=f \otimes e^{(n+1)}, \quad \gamma_{n} f=f \otimes e^{(n+1)} \otimes e^{(n+2)} \otimes \ldots
$$

By a straightforward computation one can check that

$$
L_{m} \gamma_{n}^{m}-\gamma_{n}^{m} L_{n} \text { satisfies (*). }
$$

If there exists a constant $C$ such that

$$
\prod_{1}^{n}\left\|T_{i}\right\|<C, \quad n=1,2, \ldots
$$

then $\left\|L_{n}\right\| \leqslant C$ and applying Theorem 1.1 we have

$$
\bigotimes_{1}^{\infty} T_{i} f=\lim _{m \rightarrow \infty} \gamma_{m} L_{m} \gamma_{n}^{m} f_{n}, \quad f=\gamma_{n} f_{n} .
$$

Now we shall formulate a few simple facts concerning mostly unbounded hyponormal (cohyponormal) operators. We shall use some of them in the next section. Recall that a densely defined closed operator $T$ is called cohyponormal if $T^{*}$ is hyponormal.

Proposition 1.7. For a hyponormal (cohyponormal) operator $T(W)$ the following properties hold:

(a) $\left.T^{*}\right|_{D(T)}=K T,\|K\| \leqslant 1$.

(b) If $T^{-1}$ exists, then $T^{-1}$ is also hyponormal.

(c) $\sigma(W)=\sigma_{\pi}(W)$.

(d) For any isometry $V$ the operator $V T V^{*}$ is hyponormal.

(e) If $A$ and $B$ are bounded hyponormal operators, then $A \otimes B$ is also hyponormal.

Proof. (a) The proof is similar to the one given for bounded hyponormal operators in [1].

(b) follows easily from (a).

(c) can be checked directly by the definitions.

(d) is a straightforward calculation by using the inclusion

$$
D\left(V T^{*} V^{*}\right) \supset D\left(V T V^{*}\right) \text {. }
$$

(e) We have $A \otimes B=(A \otimes I)(I \otimes B)=A_{1} B_{1}$. Therefore it is enough to show that $A_{1}, B_{1}$ are hyponormal (note that $A_{1}$ and $B_{1}$ doubly commute). Let us check the hyponormality of $A_{1}$. If $f=\sum_{k} a_{k} \otimes b_{k}$ and $b_{k}=\sum_{s} \beta_{k s} e_{s}$, where $\left(e_{s}\right)$ is an orthonormal basis, then we compute:

$$
\begin{aligned}
\left\|A_{1}^{*} f\right\|^{2} & =\left\|A_{1}^{*}\left(\sum_{k} a_{k} \otimes b_{k}\right)\right\|^{2}=\left\|\sum_{k, s} \beta_{k s} A^{*} a_{k} \otimes e_{s}\right\|^{2}=\sum_{s}\left\|\sum_{k} \beta_{k s} A^{*} a_{k}\right\|^{2} \\
& \doteq \sum_{s}\left\|A^{*} \sum_{k} \beta_{k s} a_{k}\right\|^{2} \leqslant \sum_{s}\left\|A \sum_{k} \beta_{k s} a_{k}\right\|^{2} \\
& =\left\|\sum_{k, s} A a_{k} \otimes \beta_{k s} e_{s}\right\|^{2}=\left\|\sum_{k} A a_{k} \otimes b_{k}\right\|^{2}=\left\|A_{1} f\right\|^{2} .
\end{aligned}
$$


Corollary 1.8. Let $A_{i}$ be a bounded hyponormal (cohyponormal) operator in $H_{i}$. Assume that there exist unit vectors $e_{i} \in H_{i}$ with $\sum_{i}\left\|e_{i}-A_{i} e_{i}\right\|<+\infty$. Then

$$
T=\bigotimes_{1}^{\infty} A_{i}: \underset{1\left(e_{i}\right)}{\infty} H_{i} \rightarrow \underset{1\left(e_{i}\right)}{\otimes} H_{i}
$$

is hyponormal (cohyponormal).

Proof. In fact, if we define $T_{n}=\bigotimes_{i=1}^{n} A_{i} \otimes I \otimes \ldots$, then by Proposition 1.7(e) we know that $T_{n}$ is hyponormal (the presence of infinitely many $I$ causes no problem). Since $T_{n}-T_{m}$ is also hyponormal and $T_{n} \rightarrow T$ strongly, $T_{n}^{*}$ must tend strongly to $T^{*}$. Thus $T$ is hyponormal.

We end this section by giving a few examples of unbounded hyponormal operators.

EXAMPLE 1.9. Let $\left(a_{i}\right)$ be a sequence of complex numbers such that $\left|a_{i}\right|$ is increasing. Define $T=$ weighted shift with weights $a_{i}$. Then $T$ is hyponormal. Note that in general $D(T) \neq D\left(T^{*}\right)$. By a direct computation one can check that $D(T)=D\left(T^{*}\right)$ if and only if there exists $C>0$ such that

$$
\left|a_{k}\right| \geqslant C\left|a_{k+1}\right|, \quad k=1,2, \ldots
$$

Example 1.10. Let $(C f)(x)=2^{-1 / 2}(x f(x)-d f / d x)$ be the creation operator in $L^{2}(\boldsymbol{R})$. By direct use of the Bargmann model for $C$ (or applying the results of $\left[7\right.$, p. 252]) we know that $C^{*} f(x)=2^{-1 / 2}(x f(x)+d f / d x)$, $D(C)=D\left(C^{*}\right)$ and $\left\|C^{*} f\right\| \leqslant\|C f\|$

EXAMPLE 1.11. A straightforward computation shows that $C C^{*} C$ is also hyponormal (as a weighted shift with increasing weights).

II. In case the $L_{n}$ of Theorem 1.1 are selfadjoint Marchenko has found (under condition $(*)$ ) the following nice formula:

$$
\sigma(L)=\bigcap_{n=1}^{\infty} \overline{\bigcup_{m=n}^{\infty} \sigma\left(L_{m}\right)} \quad \text { (the closure). }
$$

We shall prove an analogous formula for $\sigma_{\pi}(L)$ in terms of $\sigma_{\pi}\left(L_{n}\right)$ under certain assumptions on $L_{n}$.

THEOREM 2.1. Let $H=\lim _{y} H_{k}$. Suppose we are given a sequence $L_{n}$ of closed densely defined operators satisfying conditions (*) and (**) of Theorem 1.1. Assume that $\sigma\left(L_{m}\right)=\sigma_{n}\left(L_{m}\right)$ and

$$
\left\|\left(\lambda I-L_{m}\right)^{-1}\right\| \leqslant F_{m}\left(\dot{\operatorname{dist}}\left(\lambda, \sigma\left(L_{m}\right)\right)\right), \quad m=1,2, \ldots,
$$

where the $F_{m}(t)$ are uniformly bounded for $t \geqslant \delta>0$ (for any $\delta>0$ ). Then

$$
\sigma(L)=\sigma_{n}(L)=\bigcap_{n=1}^{\infty} \overline{\bigcup_{m=n}^{\infty} \sigma_{n}\left(L_{m}\right)} .
$$

Proof. Write $\sigma_{\pi}\left(L_{m}\right)=\sigma_{m}, \sigma_{\pi}(L)=\sigma, \bigcap_{n=1}^{\infty} \overline{\bigcup_{n=m}^{\infty} \sigma_{m}}=\sigma_{\infty}$. We have to show that $\sigma=\sigma_{\infty}$. We shall follow the ideas of [3].

a) $\sigma \supset \sigma_{\infty}$. Since $\sigma\left(L_{m}\right)=\sigma_{\pi}\left(L_{m}\right)$, we can repeat step by step the reasoning given in [3] for $L_{n}$ selfadjoint and obtain the desired inclusion.

b) $\sigma \subset \sigma_{\infty}$. We shall prove that $\boldsymbol{C} \backslash \sigma_{\infty} \subset \boldsymbol{C} \backslash \sigma$. If $\lambda \notin \sigma_{\infty}$, then there exists $n$ such that $\lambda \notin \overline{U_{m} \geqslant n} \sigma_{m}$, i.e.

$$
\operatorname{dist}\left(\lambda, \overline{\bigcup_{m \geqslant n} \sigma_{m}}\right) \geqslant \delta \quad \text { for some } \delta>0,
$$

and so $\operatorname{dist}\left(\lambda, \sigma_{m}\right) \geqslant \delta, m \geqslant n$. Hence by (3)

$$
\left\|\left(\lambda I-L_{m}\right)^{-1}\right\| \leqslant F_{m}\left(\operatorname{dist}\left(\lambda, \sigma_{m}\right)\right) \leqslant C, \quad m \geqslant n .
$$

We want to check that $\lambda \notin \sigma_{\infty}$. It is enough to check that:

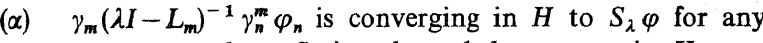
$\varphi=\gamma_{n} \varphi_{n}$, where $S_{\lambda}$ is a bounded operator in $H$.

(B) $S_{\lambda}\left(\lambda I-L_{\infty}\right) \varphi=\varphi, \varphi \in D_{\infty},\left(\lambda I-L_{\infty}\right) S_{\lambda} \psi=\psi, \psi \in H$.

Ad $(\alpha)$. Write $S_{m}=\left(\lambda I-L_{m}\right)^{-1}$. We claim that $\gamma_{m} S_{m} \gamma_{n}^{m} \varphi_{n}$ satisfies the Cauchy condition. Indeed, for $l>m \geqslant \max \left(n_{0}(\varepsilon), n\right)$ we have

$$
\begin{aligned}
\left\|\left(\gamma_{l} S_{l} \gamma_{n}^{l}-\gamma_{m} S_{m} \gamma_{n}^{m}\right) \varphi_{n}\right\| & =\left\|\left(S_{l} \gamma_{m}^{l}-\gamma_{m}^{l} S_{m}\right) \gamma_{n}^{m} \varphi_{n}\right\|=\left\|S_{l}\left(\gamma_{m}^{l} L_{m}-L_{l} \gamma_{m}^{l}\right) S_{m} \gamma_{n}^{m} \varphi_{n}\right\| \\
& \leqslant F_{l}\left(\operatorname{dist}\left(\lambda, \sigma_{l}\right)\right) \varepsilon\left(\left\|S_{m} \gamma_{n}^{m} \varphi_{n}\right\|+\left\|L_{m} S_{m} \gamma_{n}^{m} \varphi_{n}\right\|\right.
\end{aligned}
$$$$
\left.+\left\|L_{l} \gamma_{m}^{l} S_{m} \gamma_{n}^{m} \varphi_{n}\right\|\right), \quad \text { by (*) and (4). }
$$

The last expression is majorized by $\varepsilon C\left(\lambda, \varphi_{n}\right)$, as one checks directly. Hence for every $\varphi=\gamma_{n} \varphi_{n}$ the limit

$$
\lim _{m \rightarrow \infty} \gamma_{m} S_{m} \gamma_{n}^{m} \varphi_{n} \stackrel{\text { df }}{=} S_{\lambda} \varphi
$$

exists. Since $\left\|\gamma_{m} S_{m} \gamma_{n}^{m} \varphi_{n}\right\| \leqslant C\|\varphi\|, m \geqslant n$ (by (4)), we have $S_{\lambda} \in L(H)$.

Ad $(\beta)$. Let $\tilde{\varphi}=\gamma_{n} \varphi \in D_{\infty}$. Consider the sequence

$$
\varphi_{l, m}=\gamma_{l} S_{l} \gamma_{m}^{l}\left(\lambda-L_{m}\right) \gamma_{n}^{m} \varphi \text {. }
$$


Note that $\varphi_{l, m} \rightarrow \tilde{\varphi}$ as $m \rightarrow \infty$ and $l>m$. In fact, for $l>m$ $>\max \left(n_{0}(\varepsilon), n\right)$ we have

$$
\begin{aligned}
\left\|\varphi_{l, m}-\tilde{\varphi}\right\| & \leqslant F_{l}\left(\operatorname{dist}\left(\lambda, \sigma_{l}\right)\right)\left\|\left(\gamma_{m}^{l}\left(\lambda-L_{m}\right)-\left(\lambda-L_{l}\right) \gamma_{m}^{l}\right) \gamma_{n}^{m} \varphi\right\| \\
& \leqslant C\left\|\left(L_{l} \gamma_{m}^{l}-\gamma_{m}^{l} L_{m}\right) \gamma_{n}^{m} \varphi\right\| \\
& \leqslant C \varepsilon\left(\|\varphi\|+\left\|L_{l} \gamma_{m}^{l} \varphi\right\|+\left\|L_{m} \gamma_{n}^{m} \varphi\right\|\right) \leqslant C(\varphi) \varepsilon .
\end{aligned}
$$

Thus the claim is true and letting first $l \rightarrow \infty$ and next $m \rightarrow \infty$ we obtain

$$
\begin{aligned}
\varphi_{\infty, m} & =\lim _{l \rightarrow \infty} \gamma_{l} S_{l} \gamma_{m}^{l}\left(\lambda-L_{m}\right) \gamma_{n}^{m} \varphi \\
& =S_{\lambda} \gamma_{m}\left(\lambda-L_{m}\right) \gamma_{n}^{m} \varphi \rightarrow S_{\lambda}\left(\lambda-L_{\infty}\right) \tilde{\varphi} \quad \text { as } m \rightarrow \infty
\end{aligned}
$$

Hence

$$
\tilde{\varphi}=\lim _{m \rightarrow \infty} \varphi_{\infty, m}=S_{\lambda}\left(\lambda-L_{\infty}\right) \tilde{\varphi} .
$$

A similar reasoning proves that

$$
\psi_{l, m}=\gamma_{l}\left(\lambda-L_{l}\right) \gamma_{m}^{l} S_{m} \gamma_{n}^{m} \psi \rightarrow \tilde{\psi} \quad \text { as } m \rightarrow \infty,
$$

where $\tilde{\psi}=\gamma_{n} \psi$. Thus

$$
\tilde{\psi}=\lim _{m \rightarrow \infty} \psi_{\infty, m}=\lim _{m \rightarrow \infty}\left(\lambda-L_{\infty}\right) \gamma_{m} S_{m} \gamma_{n}^{m} \psi=\left(\lambda I-L_{\infty}\right) S_{\lambda} \tilde{\psi} \text {. . }
$$

COROLlary 2.2. Let $H=\lim _{\gamma} H_{k}$. Suppose we are given a sequence $T_{n}\left(W_{n}\right)$ of hyponormal (cohyponormal) operators in $H_{n}$ satisfying conditions (*) and $(* *)$ of Theorem 1.1. Then

$$
\sigma(T)=\bigcap_{n=1}^{\infty} \overline{\bigcup_{m=n}^{\infty} \sigma\left(T_{m}\right)} \quad\left(\sigma(W)=\bigcap_{n=1}^{\infty} \overline{\left.\bigcup_{m=n}^{\infty} \sigma\left(W_{m}\right)\right)} .\right.
$$

Proof. By Proposition 1.7(b), (c) we know that $\left(\lambda I-W_{m}\right)^{-1}$ is cohyponormal and $\sigma\left(W_{m}\right)=\sigma_{\pi}\left(W_{m}\right)$. On the other hand,

$$
\left\|\left(\lambda I-W_{m}\right)^{-1}\right\|=1 / \operatorname{dist}\left(\lambda, \sigma\left(W_{m}\right)\right),
$$

and so $F_{m}(r)=1 / r$ in Theorem 2.1. Since $W$ is also cohyponormal the second equality in (5) holds by Theorem 2.1. The first equality of (5) is also obvious because $T_{m}^{*}, T^{*}$ are cohyponormal and $\sigma\left(T^{*}\right)=\overline{\sigma(T)}$.

Later we shall give specific examples of applications of the above corollary. The next corollary concerns inductive limits of certain finitedimensional operators.

Let $H=\lim _{\gamma} H_{k}$ be the inductive limit of an increasing sequence of its finite-dimensional subspaces $H_{k}$, i.e. $\gamma_{n}^{n+1}$ and $\gamma_{n}$ are canonical embeddings.
Suppose we are given operators $A_{k}$ on $H_{k}$. We shall identify $A_{k}$ with $A_{k}$ $=A_{k} \oplus 0$, according to the decomposition $H=H_{k} \oplus H_{k}^{\perp}$. Let $P_{n}: H \rightarrow H_{n}$ be the orthogonal projection. Fix a uniform cross norm $\|\cdot\|_{0}$ on the set $\mathscr{F}(H)$ of finite-dimensional operators in $H$. Assume that $\|\cdot\|_{0}$ is not equivalent on $\bar{F}(H)$ to the operator norm $\|\cdot\|$. Write $r_{n}=\operatorname{dist}\left(\lambda, \sigma\left(A_{n}\right)\right)$. The following estimate has been proved in [6]:

$$
\left\|\left(\lambda-A_{n}\right)^{-1}\right\| \leqslant 3 r_{n}^{-1} \exp \left[39\left\|A_{n}\right\|_{0} r_{n}^{-1} \tau\left(r_{n} /\left(6\left\|A_{n}\right\|_{0}\right)\right)\right],
$$

where $\tau$ is a decreasing function in $(0, \infty)$ depending on $\left\|P_{n}\right\|_{0}$. Applying Theorem 2.1 we have

Corollary 2.3. Let $H, H_{n}, A_{n},\|\cdot\|_{0}$ be as above. Suppose that the sequence $A_{n}$ satisfies (*) of Theorem 1.1 and there exists $C>0$ such that $\left\|A_{n}\right\|_{0} \leqslant C, n=1,2, \ldots$ Then

$$
\sigma(A)=\bigcap_{n=1}^{\infty} \overline{\bigcup_{m=n}^{\infty} \sigma\left(A_{m}\right)}
$$

where $A=\lim _{\rightarrow} A_{n}$.

Proof. Since $\left\|A_{n}\right\|_{0} \leqslant C, n=1,2, \ldots$, by (6) we can put in Theorem 2.1

$$
F_{n}(r)=3 r^{-1} \exp \left[39\left\|A_{n}\right\|_{0} r^{-1} \tau\left(r /\left(6\left\|A_{n}\right\|_{0}\right)\right)\right] \text {. }
$$

In the next section we shall give an example of application of Corollary 2.3 to trace class operators.

III. This section contains a few applications of the results of the previous one.

a) Let $L$ be a trace class operator in a separable Hilbert space $H$. Suppose that there exists an orthonormal basis $\left(e_{k}\right)$ in which $L$ can be written as a tridiagonal matrix $a_{i j}=\left(L e_{j}, e_{i}\right)$. Let $H_{n}=\operatorname{span}\left(e_{1}, \ldots, e_{n}\right)$ and denote by $P_{n}$ the orthogonal projection on $H_{n}$. Define $L_{n}$ to be the compression of $L$ to $H_{n}$, i.e. $L_{n}=P_{n} L P_{n}$. Let $\gamma_{n}^{n+1}: H_{n} \rightarrow H_{n+1}, \gamma_{n}: H_{n} \rightarrow H$ be the inclusion embeddings. If $\varphi=\sum_{1}^{n} \varphi_{i} e_{i}$, then by a direct computation we find

$$
\left\|\left(L_{m} \gamma_{n}^{m}-\gamma_{n}^{m} L_{n}\right) \varphi\right\|=\left|a_{n+1, n} \varphi_{n}\right|, \quad m>n .
$$

Since $L$ is of trace class, $a_{n+1, n} \rightarrow 0$ as $n \rightarrow \infty$.

Now choose the cross norm $\|T\|_{0}=\operatorname{tr}\left(T T^{*}\right)^{1 / 2}$. Since $L_{m} \rightarrow L$, there exists $M>0$ such that $\left\|L_{m}\right\|_{0} \leqslant M, m=1,2, \ldots$ On the other hand, (7) and the convergence to zero of $a_{n+1, n}$ imply condition $(*)$ of Theorem 1.1 Applying Corollary 2.3 we obtain the (surely known) 
Proposition 3.1. Let $L$ be a tridiagonal trace class operator and let $L_{m}$ be as above. Then

$$
\sigma(L)=\bigcap_{n=1}^{\infty} \overline{\bigcup_{m=n}^{\infty} \sigma\left(L_{m}\right)} .
$$

Remark 3.2. Note that for a tridiagonal operator $L=\left(a_{i j}\right)$ the convergence of

$$
\sum_{k}\left|a_{k k}\right|, \quad \sum_{k}\left|a_{k+1, k}\right|, \quad \sum_{k}\left|a_{k, k+1}\right|
$$

guarantees that $L$ is of trace class.

$\beta$ ) The next application concerns cohyponormal operators. Suppose we are given a sequence of cohyponormal operators $T_{i}$ in $H_{i}$. Assume that there exist $e_{i} \in H_{i},\left\|e_{i}\right\|=1$, with

$$
\sum_{i}\left\|T_{i}^{*} e_{i}-e_{i}\right\|<+\infty
$$

Then as we know $\bigotimes_{1}^{\infty} T_{i}: \bigotimes_{1\left(e_{i}\right)}^{\infty} H_{i} \rightarrow \bigotimes_{1\left(e_{i}\right)}^{\infty} H_{i}$ is also cohyponormal (by Corollary 1.8 adapted for arbitrary cohyponormal operators). In order to find $\sigma\left(\otimes_{i} T_{i}\right)$ we apply Corollary 2.2. Namely, we define

$$
K_{n}=\bigotimes_{1}^{n} H_{i}, \quad L_{n}=\bigotimes_{1}^{n} T_{i}, \quad \gamma_{n}^{n+1}: K_{n} \ni f \rightarrow f \otimes e_{n+1} \in K_{n+1},
$$

and we obtain

Proposition 3.3. Let $T_{i}$ be a sequence of cohyponormal operators in $H_{i}$. If there exists a sequence $e_{i} \in D\left(T_{i}^{*}\right)$ of unit vectors such that (8) is satisfied, then

$$
\therefore \quad \sigma\left(\bigotimes_{1}^{\infty} T_{i}\right)=\bigcap_{n=1}^{\infty} \overline{\bigcup_{m=n}^{\infty} \sigma\left(L_{m}\right),}
$$

where $L_{m}=\bigotimes_{1}^{m} T_{i}$.

Proof. By (8) and direct computation one can check that for any $\varepsilon>0$ there exists $n_{0}=n_{0}(\varepsilon)$ such that for $m>n \geqslant n_{0}$ and $\varphi_{n} \in D\left(L_{n}\right)$

$$
\left\|\left(L_{n} \gamma_{n}^{m}-\gamma_{n}^{m} L_{n}\right) \varphi_{n}\right\| \leqslant \varepsilon\left\|L_{n} \varphi_{n}\right\| .
$$

Thus condition (*) of Theorem 1.1 holds. The same computation gives an analogous inequality for $L_{n}^{*}$ and so condition (**) also holds. Applying Corollary 2.2 gives the desired result.

Remark 3.4. In case the $T_{i}$ are bounded and $\prod_{1}^{\infty}\left\|T_{i}\right\|<+\infty$ it is - enough to assume that $\sum_{i}\left\|e_{i}-T_{i} e_{i}\right\|<+\infty$ instead of (8).

Here are two immediate applications of Proposition 3.3. a) Suppose we are given cohyponormal operators $S_{k} \in L\left(H_{k}\right)$ such that $\left\|S_{k}\right\| \leqslant C, k=1,2, \ldots$ Let $\operatorname{Exp} S_{k}$ be the exponent of $S_{k}$ in $\operatorname{Exp} H_{k}$ (see [2] for the definitions). Since $\operatorname{Exp}\left(\oplus_{1}^{n} S_{k}\right)=\otimes_{1}^{n} \operatorname{Exp}_{k}$ we have

(9) $\quad \prod_{1}^{n}\left\|\operatorname{Exp} S_{k}\right\|=\left\|\bigotimes_{1}^{n} \operatorname{Exp} S_{k}\right\|=\left\|\operatorname{Exp}\left(\bigoplus_{1}^{n} S_{k}\right)\right\| \leqslant \exp \left\|\bigoplus_{1}^{n} S_{k}\right\| \leqslant e^{c}$.

Let $1=1 \oplus 0 \oplus \ldots \in \operatorname{Exp} H_{k}$. Then $\operatorname{Exp} S_{k} 1=1$. Hence by (9) one can define the (bounded) operator

$$
S=\bigotimes_{1}^{\infty} \operatorname{Exp} S_{k}: \bigotimes_{1(1)}^{\infty} \operatorname{Exp} H_{k} \rightarrow \bigotimes_{1(1)}^{\infty} \operatorname{Exp} H_{k} .
$$

Note that $S=\operatorname{Exp} \oplus_{1}^{\infty} S_{k}$.

Now we put $T_{k}=\operatorname{Exp} S_{k}$ and applying Proposition 3.3 we have

$$
\sigma(S)=\bigcap_{n=1}^{\infty} \overline{\bigcup_{m=n}^{\infty} \sigma\left(S_{1}\right) \ldots \sigma\left(S_{m}\right)}
$$

where $\sigma\left(S_{1}\right) \ldots \sigma\left(S_{m}\right)=\left\{\lambda: \lambda=\lambda_{1} \ldots \lambda_{m}, \lambda_{k} \in \sigma\left(S_{k}\right)\right\}$.

b) Let $d \mu=(2 \pi)^{-1 / 2} \exp \left(-x^{2} / 2\right) d x$ be the Gaussian measure on $R$ and let $0<|\alpha| \leqslant 1, \alpha \in \boldsymbol{R}$. Following Mlak we define the operator $C_{\alpha}$ in $L^{2}(\mu)$ by $\left(C_{x} f\right)(x)=f(\alpha . x)$. It turns out that $C_{x}$ is bounded and $\sigma\left(C_{x}\right)$ $=\left\{z:|z| \leqslant|\alpha|^{-1 / 2}\right\}$ (see [5]). Moreover, for any sequence $\left(\alpha_{i}\right), 0<\left|\alpha_{i}\right| \leqslant 1$, such that $c=\prod_{1}^{\infty}\left|\alpha_{i}\right|^{-1 / 2}<+\infty$ Mlak defined the operator

$$
\bigotimes_{1}^{\infty} C_{\alpha_{i}}: \bigotimes_{1(1)}^{\infty} L^{2}(\mu) \rightarrow \bigotimes_{1(1)}^{\infty} L^{2}(\mu) .
$$

It can be identified with the operator

$$
C_{\left(\alpha_{i}\right)}: L^{2}(\tilde{\mu}) \rightarrow L^{2}(\tilde{\mu})
$$

given by $\left(C_{\left(\alpha_{i}\right)} f\right)(x)=f\left(\alpha_{1} x_{1}, \alpha_{2} x_{2}, \ldots\right)$, where $\tilde{\mu}=\otimes_{1}^{\infty} d \mu$ is the Gaussian measure on the Borel subsets of $\boldsymbol{R}^{\infty}$. By a direct computation we check that the $C_{\alpha_{i}}$ are cohyponormal. Hence applying Proposition 3.3 (see Remark 3.4) we have

$$
\sigma\left(C_{\left(a_{i}\right)}\right)=\bigcap_{n=1}^{\infty} \overline{\bigcup_{m=n}^{\infty} \sigma\left(L_{m}\right)}
$$

where $L_{n}=\bigotimes_{1}^{n} C_{\alpha_{i}}$. Since $\sigma\left(L_{n}\right)=\left\{z: z=z_{1} \ldots z_{n},\left|z_{k}\right| \leqslant\left|\alpha_{k}\right|^{-1 / 2}\right\}$, by (10) we . have

$$
. \sigma\left(C_{\left(\alpha_{i}\right)}\right)=\{z:|z| \leqslant c\} .
$$


IV. Concluding comments and results. First note the following remarks.

Remark 4.1. Theorem 2.1 remains true for inductive limits of linear mappings $L_{n}: E_{n} \rightarrow F_{n}$ between Banach spaces under the same assumptions on $L_{n}$ as before.

Remark 4.2. One can also consider inductive limits of $C^{*}$-algebras $A_{k}$ with *-monomorphisms $\gamma_{k}^{k+1}: A_{k} \rightarrow A_{k+1}$ and continuous operators $L_{k}: A_{k}$ $\rightarrow A_{k}$ satisfying conditions analogous to the previous ones. In particular, by applying this procedure to Calkin algebras $A_{n}=A\left(H_{n}\right)$ (over Hilbert spaces $H_{n}$ ) and $L_{n} a=b_{n} a$ one obtains a result corresponding to Theorem 2.1 or its corollaries. The details are left to the interested reader.

Now we shall extend Theorem 2.1 to commuting tuples of normal operators. In order to do this we first modify slightly the form of conditions $(*)$ and $(* *)$ using the notion of bounded vector. Recall that for a densely defined operator $A$ a vector $f$ is said to be a bounded vector for $A$ if there are $c_{f}>0$ and $M_{f}>0$ such that

$$
\left\|A^{k} f\right\| \leqslant M_{f} c_{f}^{k}, \quad k=1,2, \ldots
$$

Denote by $B(A)$ the set of all bounded vectors for $A$ and by $B_{c}(A)$ the set of all those $f \in B(A)$ which satisfy (B) with $c_{f}=c$. It is clear that

$$
B(A)=\bigcup_{k=1}^{\infty} B_{k}(A)
$$

Now let $H=\lim _{\gamma} H_{k}$ and let $L_{k}$ be a sequence of closed densely defined operators in $H_{k}$. The sequence $L_{k}$ is said to satisfy $(*)_{1}$ or $(* *)_{1}$, respectively, if $(*)$ or $(* *)$ hold for $L_{k}$ and vectors in $B\left(L_{k}\right)$ or $B\left(L_{k}^{*}\right)$, respectively. It turns out that for normal operators, under certain natural assumptions on $\gamma_{n}^{m}$, condition $(*)_{1}$ implies $(* *)_{1}$. Namely, we have

LemMa 4.3. Let $N_{k}$ be a sequence of normal operators in $H_{k}$. Suppose that $\forall_{c>0} \quad \exists_{c_{1}} \geqslant c$ such that

$$
\gamma_{n}^{m} B_{c}\left(N_{n}\right) \subset B_{c_{1}}\left(N_{m}\right), \quad \forall_{m \geqslant n} .
$$

If the sequence $N_{k}$ satisfies $(*)_{1}$, then it satisfies $(* *)_{1}$.

Proof. We shall suitably modify the method used by Rosenblum in his proof of the Putnam-Fuglede theorem [8]. In fact, we shall use (and prove) a slightly modified form of $(*)_{1}$ (and $\left.(* *)_{1}\right)$. Namely, suppose that

$$
\forall_{\varepsilon>0} \exists_{n_{0}} \forall_{m>n \geqslant n_{0}} \forall_{f \in B_{c}\left(N_{n}\right)} \quad\left\|\left(N_{m} \gamma_{n}^{m}-\gamma_{n}^{m} N_{n}\right) f\right\| \leqslant \varepsilon(1+c)\|f\| .
$$

By the above assumptions and induction we have

$\left\|\left(N_{m}^{k} \gamma_{n}^{m}-\gamma_{n}^{m} N_{n}^{k}\right) f\right\| \leqslant k \varepsilon c_{1}^{k}(1+c)\|f\|, \quad k=1,2, \ldots, f \in B_{c}\left(N_{n}\right), m>n \geqslant n_{0}$
Hence by a direct computation we have for $m>n \geqslant n_{0}$

$$
\left\|\left(e^{i \bar{i} N_{m}} \gamma_{n}^{m}-\gamma_{n}^{m} e^{i \lambda N_{n}}\right) f\right\| \leqslant \varepsilon|\lambda| c_{1}(1+c) e^{|\lambda| c_{1}}\|f\|, \quad \lambda \in C, f \in B_{c}\left(N_{n}\right) .
$$

Similarly one can check that

(11) $\left\|e^{i \lambda N_{m}^{*}}\left(e^{i \lambda N_{m}} \gamma_{n}^{m}-\gamma_{n}^{m} e^{i \bar{\lambda} N_{n}}\right) e^{-i \bar{\lambda} N_{n}} e^{-i \lambda N_{n}^{s}} f\right\|$

$$
\leqslant \varepsilon e^{|\lambda| c_{1}}(1+c)|\lambda| c_{1} e^{|\lambda|\left(c_{1}+2 c\right)}\|f\|, \quad m>n \geqslant n_{0}, f \in B_{c}\left(N_{n}\right) .
$$

Since $e^{a N_{k}+b N_{k}^{*}}=e^{a N_{k}} e^{b N_{k}^{*}}, a, b \in C, e^{i\left(\bar{\lambda} N_{m}+\lambda N_{m}^{*}\right\}}$ is unitary and by (11) we have

$$
\left\|e^{i \lambda N_{m}^{*}} \gamma_{n}^{m} e^{-i \lambda N_{n}^{*}} f\right\| \leqslant\|f\|+\varepsilon c_{2}(|\lambda|)\|f\|,
$$

where $c_{2}(|\lambda|)=e^{|\lambda| c_{1}}(1+c)|\lambda| c_{1} e^{|\lambda|\left(c_{1}+2 c\right)}$

Let $F_{f}^{m n}(\lambda)=e^{i \lambda N_{m}^{*}} \gamma_{n}^{m} e^{-i \lambda N_{n}^{*}} f$ be an entire vector-valued function, where $f \in B_{c}\left(N_{n}\right)$. Write $C=\{z \in C:|z|=r\}$. Then by the Cauchy formula (we omit the indices $m, n)$

$$
F_{f}^{\prime}(0)=\frac{1}{2 \pi i} \int_{C} \frac{F(u)}{u^{2}} d u
$$

Hence

$$
\left\|F_{f}^{\prime}(0)\right\| \leqslant \frac{1}{r} \max \left|F_{f \mid=r}(z)\right|,
$$

and so by (12) we have

$$
\left\|F_{f}^{\prime}(0)\right\| \leqslant \frac{1}{r}\left(1+\varepsilon c_{2}(r)\right)\|f\| .
$$

But $F_{f}^{\prime}(0)=i\left(N_{m}^{*} \gamma_{n}^{m}-\gamma_{n}^{m} N_{n}^{*}\right) f$, so

$$
\left\|\left(N_{m}^{*} \gamma_{n}^{m}-\gamma_{n}^{m} N_{n}^{*}\right) f\right\| \leqslant\left(1 / r+\varepsilon c_{2}(r) / r\right)\|f\| .
$$

Now for any $\eta>0$ put $r=2 / \eta$ and take $\varepsilon=\varepsilon(\eta)$ so small that $\varepsilon c_{2}(r) / r \leqslant \eta / 2$. It follows (by (13)) that

$$
\left\|\left(N_{m}^{*} \gamma_{n}^{m}-\gamma_{n}^{m} N_{n}^{*}\right) f\right\| \leqslant \eta\|f\|
$$

for $m>n \geqslant n_{0}(\varepsilon)$ and $f \in B_{c}\left(N_{n}\right)$. Thus $(* *)_{1}$ holds.

Corollary 4.4. Let $H=\lim _{\rightarrow} H_{k}$ and let $N_{k}$ be a sequence of normal operators satisfying $(*)_{1}$. If $\gamma_{n}^{m} B_{c}\left(N_{n}\right) \subset B_{c_{1}}\left(N_{m}\right), \forall_{m>n}$, then the operator $N=\bar{N}_{\infty}$ is normal in $H\left(N_{\infty}\right.$ is the same as in Theorem 1.1).

Proof. By Lemma 4.3, $(* *)_{1}$ holds for $N_{k}$ so it is obvious that $\gamma_{m} N_{m}^{*} \gamma_{n}^{m} \varphi_{n}$ tends to $N^{*} \varphi$ as $m \rightarrow \infty$, where $\varphi=\gamma_{n} \varphi_{n}, \varphi_{n} \in B\left(N_{n}\right)$. Let $\bigcup_{k} \gamma_{k} B\left(N_{k}\right)=X$. As we know $D\left(N_{\infty}\right) \subset D\left(N_{\infty}^{*}\right)$ (see the proof of Th. 1.1). It 
is also clear that $\left\|N_{\infty} f\right\|=\left\|N_{\infty}^{*} f\right\|$ for $f \in D\left(N_{\infty}\right)$. Moreover, $X$ is dense in $D\left(N_{x}\right), N_{x} X \subset X, N_{\infty}^{*} X \subset X$, so $\bar{N}_{\infty}=N$ must be normal (see [11]).

Now we shall extend Theorem 2.1 to commuting normal operators. Suppose we are given a sequence of normal commuting operators $N_{1 k}, \ldots, N_{s k}$ in $H_{k}$, i.e. $N_{p k}=\int \lambda d E_{p k}$ and the spectral measures $E_{p k}$ pairwise commute. By the joint spectrum $\sigma\left(N_{1 k}, \ldots, N_{s k}\right)$ we mean the joint approximate point spectrum of $N_{1 k}, \ldots, N_{s k}$ (see [10] for the definition). Let $H=\lim _{\vec{\gamma}} H_{k}$. Write $B_{c}\left(N_{k}\right)=B_{c}\left(N_{1 k}\right) \cap \ldots \cap B_{c}\left(N_{s k}\right)$ and suppose that $\forall c>0 \vec{\exists}_{c_{1} \geqslant c}$ such that

$$
\gamma_{n}^{m} B_{c}\left(N_{n}\right) \subset B_{c_{1}}\left(N_{m}\right)
$$

The system $N_{k}=\left(N_{1 k}, \ldots, N_{s k}\right)$ is said to satisfy condition $(*)_{1}$ if

$$
\forall_{\varepsilon>0} \exists_{n_{0}(\varepsilon)} \forall_{m>n \geqslant n_{0}(\varepsilon)} \quad\left\|\left(N_{p m} \gamma_{n}^{m}-\gamma_{n}^{m} N_{p n}\right) f\right\| \leqslant \varepsilon(1+c)\|f\|,
$$

for any $f \in B_{c}\left(N_{n}\right), p=1, \ldots, s$.

In what follows we shall restrict ourselves to commuting pairs $(s=2)$ of normal operators, but the results hold for any $s$.

THEOREM 4.5. Let $H=\lim _{\vec{\gamma}} H_{k}$ and suppose we are given a sequence $A_{m}, B_{m}$ of commuting normal operators in $H_{m}$. If $\gamma_{n}^{m}$ satisfies (14) for any $m \geqslant n$ and $\left(A_{m}, B_{m}\right)$ satisfy $(*)_{1}$, then

$$
\sigma(A, B)=\bigcap_{m=1}^{\infty} \overline{\bigcup_{m=n}^{\infty} \sigma\left(A_{n}, B_{n}\right)}
$$

where $A=\bar{A}_{\infty}, B=\bar{B}_{\infty}$.

Proof. Denote bx $\sigma_{\infty}$ the right-hand side of (15).

a) The inclusion $\sigma(A, B) \supset \sigma_{\infty}$ can be proved in the same way as the corresponding inclusion in the proof of Theorem 2.1 (note that $A$ and $B$ are normal by Corollary 4.4).

b) The opposite inclusion $\sigma_{\infty} \supset \sigma(A, B)$ may be proved as follows. If $\lambda=\left(\lambda_{1}, \lambda_{2}\right) \notin \sigma_{\infty}$, then for some $\delta>0$ and $n$

$$
\operatorname{dist}\left(\lambda, \sigma\left(A_{m}, B_{m}\right)\right)>\delta, \quad \forall_{m \geqslant n} .
$$

Now writing $A_{\lambda m}=\lambda_{1} I-A_{m}, B_{\lambda m}=\lambda_{2} I-B_{m}$ we see that $A_{\lambda m}^{*} A_{\lambda m}+B_{\lambda m}^{*} B_{\lambda m}$ is invertible, i.e. there exists $R_{\lambda m} \in L\left(H_{m}\right)$ such that

$$
R_{\lambda m}\left(A_{\lambda m}^{*} A_{\lambda m}+B_{\lambda m}^{*} B_{\lambda m}\right) f=f
$$

for $f$ in the domain of $A_{\lambda m}^{*} A_{\lambda m}+B_{\lambda m}^{*} B_{\lambda m}$. Since $A_{m}, B_{m}$ are commuting and normal,

$$
\sigma\left(A_{\lambda m}^{*} A_{\lambda m}+B_{\lambda m}^{*} B_{\lambda m}\right)=\left\{\left|z_{1}\right|^{2}+\left|z_{2}\right|^{2}:\left(z_{1}, z_{2}\right) \in \sigma\left(A_{\lambda m}, B_{\lambda m}\right)\right\} .
$$

It follows that

$$
\inf _{m \geqslant n} \inf _{\{}\left\{\left|z_{1}\right|^{2}+\left|z_{2}\right|^{2}:\left(z_{1}, z_{2}\right) \in \sigma\left(A_{\lambda m}, B_{\lambda m}\right)\right\}=\inf _{m \geqslant n} \operatorname{dist}\left(\lambda, \sigma\left(A_{m}, B_{m}\right)\right) \geqslant \delta
$$

by (16). Thus

$$
\text { We claim that } \lambda \notin \sigma(A, B) \text {. In fact, by (17) and (+) we have }
$$

$$
\left(\left(A_{\lambda m}^{*} A_{\lambda m}+B_{\lambda m}^{*} B_{\lambda m}\right) f, f\right) \geqslant \delta\|f\|
$$

so letting $m \rightarrow \infty$ we obtain the desired claim.

Remark 4.6. The following reasoning shows that Theorem 4.5 cannot be extended further (even to bounded commuting subnormal pairs). In fact, by the result of [9] there exists a sequence of commuting bounded subnormal pairs $T_{k}$, $W_{k}$ in certain $H_{k}$ such that

$$
\sigma\left(\stackrel{\oplus}{1}_{1}^{\infty} T_{k}, \stackrel{\oplus}{1}_{W_{k}}\right) \neq \overline{\bigcup_{1}^{\infty} \sigma\left(T_{k}, W_{k}\right)}
$$

where $\sigma\left(T_{k}, W_{k}\right)$ stands for the Taylor joint spectrum. Obviously

$$
\stackrel{\oplus}{1}_{1}^{\infty} H_{k}=\lim _{\rightarrow} \underset{1}{\stackrel{s}{\oplus}} H_{k}
$$

where

$$
\begin{gathered}
\gamma_{n}^{n+1}:\left(x_{1}, \ldots, x_{n}\right) \rightarrow\left(x_{1}, \ldots, x_{n}, 0\right), \\
\gamma_{n}:\left(x_{1}, \ldots, x_{n}\right) \rightarrow\left(x_{1}, \ldots, x_{n}, 0,0, \ldots\right) .
\end{gathered}
$$

Let $L_{n}=\oplus_{1}^{n} T_{k}, M_{n}=\oplus_{1}^{n} W_{k}$. Then $L_{n+1} \gamma_{n}^{n+1}-\gamma_{n}^{n+1} L_{n}=0, M_{n+1} \gamma_{n}^{n+1}$ $-\gamma_{n}^{n+1} M_{n}=0$. If we had for $L=\bigoplus_{1}^{\infty} T_{k}, M=\bigoplus_{1}^{\infty} W_{k}$ the equality

$$
\sigma(L, M)=\bigcap_{n=1}^{\infty} \overline{\bigcup_{s=n}^{\infty} \sigma\left(L_{s}, M_{s}\right)}
$$

then we would have

$$
\sigma(L, M)=\bigcap_{n=1}^{\infty} \overline{\bigcup_{s=n}^{\infty} \sigma\left(L_{s}, M_{s}\right)}=\bigcap_{n=1}^{\infty} \overline{\bigcup_{k=1}^{\infty} \sigma\left(T_{k}, W_{k}\right)}=\overline{\bigcup_{k=1}^{\infty} \sigma\left(T_{k}, W_{k}\right)}
$$

and this contradicts $(\alpha)$. 
[1] K. Clancey, Seminormal Operators, Lecture Notes in Math. 742, Springer, 1979.

2] R. L. Dobrushin and R. A. Minlos, Polynomials of linear random functions, Uspekhi Mat. Nauk 32 (2) (194) (1977), 67-122 (in Russian).

[3] A. V. Marchenk o, Selfadjoint differential operators with an infinite number of independent variables, Mat. Sb. 96 (2) (1975), 276-293 (in Russian).

[4] W. Mlak, Introduction to the Theory of Hilbert Spaces, PWN, Warszawa 1982 (in Polish).

[5] -, Operators induced by change of Gaussian variables, Ann. Polon. Math., to appear.

[6] A. Pokrzywa, On continuity of spectra in norm ideals, Linear Algebra Appl. 69 (1985), 121-130.

[7] M. Reed and B. Simon, Methods of Modern Mathematical Physics II: Fourier Analysis, Self-adjointness, Academic Press, New York 1975.

[8] M. Rosenblum, On a theorem of Fuglede and Putnam, J. London Math. Soc. 33 (1958), 376-377.

[9] J. K. Rudol, The spectrum of orthogonal sums of subnormal pairs, preprint, 1985.

[10] Yu. S. Samoilenko, Spectral Theory of Systems of Selfadjoint Operators, Naukova Dumka, Kiev 1984

[11] J. Stochel and F. Szafraniec, Bounded vectors and formally normal operators, Operator Theory: Adv. Appl. 11 (1983), 363-370.

[12] Y. Yamasaki, Kolmogorov's extension theorem for infinite measures, Publ. RIMS Kyoto Univ. 10 (1975), 381-411.

INSTYTUT MATEMATYCZNY POLSKIEJ AKADEMII NAUK

ODDZIAL W KRAKOWIE

INSTITUTE OF MATHEMATICS, POLISH ACADEMY OF SCIENCES

KRAKÖW BRANCH

Solskiego 30, 31-027 Kraków, Poland

\section{Groups of isometries on operator algebras}

by

\section{STEEN PEDERSEN (Aarhus)}

Abstract. Let $\varrho$ be a $C_{0}$-group of isometries on a unital $C^{*}$-algebra $A$. If $u(t)=\varrho(t) 1$ and $\alpha(t) a=u(t)^{*} \varrho(t) a$, then $\varrho(t) a=u(t) \alpha(t) a, \alpha$ is a $C_{0}$-group of $*$-automorphisms on $A$ and $u$ is a unitary 1-cocycle. We study this decomposition of $\varrho$; as a consequence we obtain a classification of the generators of $C_{0}$-groups of isometries on $A$.

Introduction. In [18] Kadison proved that an isometry of a unital $C^{*}$-algebra $A$ onto itself can be decomposed into a $C^{*}$-homomorphism followed by multiplication by a unitary. We study the consequences of applying this decomposition to a strongly continuous isometric representation $\varrho$ of a topological group on $A$. We prove that the $C^{*}$-homomorphic part of $\varrho$ is a strongly continuous group of $*$-automorphisms and that $\varrho$ is norm-continuous if $A$ is a von Neumann algebra. We establish conditions, global as well as local, which are satisfied by $\varrho$ if and only if it is a group of $*$-automorphisms.

Using perturbation theory for *-automorphism groups we prove that if $\varrho$ is a one-parameter group of isometries on $A$ with generator $\delta$, then there exist $(\gamma, v, h)$, where $\gamma$ is the generator of a one-parameter group of *-automorphisms on $A, v$ is a unitary in $A$ and $h$ is a selfadjoint element of $A$, such that $\mathscr{D}(\delta)=v^{*} \mathscr{D}(\gamma)$ and

$$
\delta(a)=v^{*} \gamma(v a)+i v^{*} h v a
$$

for $a$ in $\zeta(\delta)$. Using this we give local and global conditions equivalent to the fact that the unitary part of $\varrho$ is a group.

In the next part of the paper we specialize to the case where $\varrho$ is a one-parameter group. We observe that in some representations of $A, \varrho(t) a$ $=u(t) a v(t)$, where $u$ and $v$ are strongly continuous unitary groups. We study the generators of (semi-) groups of this form.

This study was motivated by applications to quantum mechanics (e.g. [15], [22], [25]) and partially inspired by the corresponding problems for a one-parameter semigroup on a Hilbert space if each element of the semigroup is polar decomposed [11], [12]. 